\title{
A Consideration of the Decision to Reallocate Educational Resources on the Basis of a Comparison of Academic Area Test Scores across Schools
}

\author{
Melvin V. Borland, Roy M. Howsen \\ Department of Economics, Western Kentucky University, Bowling Green, USA. \\ Email: roy.howsen@wku.edu \\ Received July $30^{\text {th }}, 2010$; revised September $25^{\text {th }}, 2010$; accepted September $30^{\text {th }}, 2010$.
}

\begin{abstract}
Although suggestions for the reallocation of educational resources for individual schools or school districts are often made on the basis of comparative test scores by academic area across such individual schools or school districts, such scores, it is shown, are neither necessarily nor sufficiently informative for the systematic determination of utility increasing reallocations of those resources. To the extent that different administrators hold different educational utility functions in terms of test scores, then, even under assumptions of concurrent, but individual utility maximizations, different distributions of educational resources would be expected. Students in individual school or school districts, therefore, would be expected to have comparative test scores that are necessarily low in at least one academic area and high in at least one other, mutatis mutandis. Reallocations made on the basis of comparative test scores by academic area within individual schools or school districts, therefore, cannot, it is shown, be expected to systematically increase educational utility.
\end{abstract}

Keywords: Reallocation Resources, Test Scores

Comparative test scores by academic area for individual schools or school districts are extensively reported by the various media and the subject of much discussion by policy makers, educational authorities, and members of the general public. ${ }^{1}$ It is on the basis of such comparative scores across schools or districts that reforms in resource allocation are suggested. The typical suggestion given the report of comparative test scores by academic area is to use relatively more resources in the academic area(s) associated with the relatively low comparative test score(s) and, therefore, necessarily fewer resources in the academic area(s) associated with the relatively high comparative test score(s), given that the availability of such resources is constrained. Is such a change in resource allocation necessarily educational utility-increasing (Leithwood \& Stager, 1989; Simon, 1976)? $?^{2}$ For the purpose of stimulating empirical work on the relationship between comparative test scores and resource allocation, consider the following model of optimal resource allocation and the discussion of exogenous effects given optimal resource allocation.

\section{Optimal Resource Allocation}

The educational utility function considered in this paper is limited to the scores, $q$ and $v$, associated respectively with the areas of quantitative reasoning and verbal proficiency, only. This limitation on the number of areas for which test scores are associated allows us with ease of exposition to concentrate on the particular variables in question in this model of optimal resource allocation, that is, on the amount of resources, $r_{q}$, used in the production of quantitative reasoning and the amount of resources, $r_{v}$, used in the production of verbal proficiency and results in no loss of generality.
Rational school administrators under these conditions are expected to maximize educational utility, $u$, as a function, $u$, of $q$ and $v$, i.e., to maximize

$$
u=u(q, v),
$$

subject to

$$
R=r_{q}+r_{v}
$$

In Equation (2) above, $R$ is the sum of the number of units of resources distributed to the areas of quantitative reasoning and verbal proficiency, that is, of $r_{q}$ and $r_{v}$, respectively. ${ }^{3}$ The budget constraint for the school administrator is implied by the finite number of units of resources available for distribution.

The absolute test scores associated with the areas of quantitative reasoning and verbal proficiency are assumed to be related to the quantities of resources, $r_{q}$ and $r_{v}$, by production processes, $q$ and $v,{ }^{4}$ respectively. The proposition that time, at least, correlates positively with measured achievement is supported by a considerable literature. ${ }^{5}$ (Levin \& Tang, 1987; Roberts et al, 1986; Karweit, 1984; Penham \& Lieberman, 1980; Fisher \& Filby, 1929; Wiley \& Harnischfeger, 1974; Carroll, 1963)

By substitution, where the production processes, $q$ and $v$, in which the school administrator has the finite number of units of resources available for distribution state the levels of educational achievements by area as functions of $r_{q}$ and $r_{v}$, respectively, Equation (1) can be rewritten as

$$
u=u\left(q\left(r_{q}\right), v\left(r_{v}\right)\right) .
$$

Form the Lagrange function,

$$
u=u\left(q\left(r_{q}\right), v\left(r_{v}\right)\right)-\left(R_{f}-r_{q}-r_{v}\right),
$$

where $\lambda$ is as yet an undetermined multiplier, to solve for the 
optimal quantities of $r_{q}$ and $r_{v}$. The 1st-order conditions for the maximization of educational utility are determined from the solution of the three partials of Equation (4) with respect to $r_{q}$, $r_{v}$, and $\lambda$, each set equal to 0 . As such, the utility-maximizing conditions require the distribution of available resources so that

$$
u r_{q}=u r_{v}{ }^{6}
$$

These 1st-order conditions require that the ratios of the marginal utilities of the last unit of resources spent on quantitative reasoning and the last unit of resources spent on verbal proficiency be equal to the ratio of their associated prices. ${ }^{7}$ In this case, the associated prices are in terms of units of resources and are, therefore, each equal to 1 . These conditions imply that, if the increase in utility that would result from spending an additional unit of educational resources on either quantitative reasoning or verbal proficiency was greater than that which would result from spending an additional unit on the other, utility could be increased if and only if resources are reallocated to that area with the larger marginal utility and, since $\mathrm{R}$ is a finite number of units, necessarily from that area with the smaller marginal utility. The multiplier, $\lambda$, is interpreted, in this case, as the marginal educational utility of resources, that is, as the change in educational utility with respect to a change in the finite number of units of resources available for distribution. ${ }^{8}$

\section{Exogenous Effects Given Optimal Resource Allocations}

If the allocation of available resources for an individual school or school district satisfies Equation (5), levels of achievement associated with the various areas of testing, $q$ and $v$, are implied by production processes, $q$ and $v$, and educational utility is maximized. Comparative area scores for that individual school or school district associated with such utility-maximizing levels of academic achievement, however, are dependent not only on levels of the absolute achievement of students in that individual school or school district, but on levels of the absolute achievement of students in other individual schools or school districts to which comparisons are made. And such other levels of absolute achievement are implied by other, although nevertheless optimal, allocations of educational resources with respect to the associated utility functions of the other individual schools or school districts.

There may be several explanations, under such a comparison, for the existence of different allocations of educational resources across individual schools or school districts, even under assumptions of concurrent, but individual utility maximization. One such explanation is the existence of different educational utility functions across such individual schools or school districts to which different allocations are made. Given the educational utility function for an individual school or school district, suppose that the allocation of resources satisfies Equation (5). Note that, even if the school administrator of the individual school or school district is the only school administrator to do so with respect to the educational utility function attached to that individual school or school district, that is, to allocate resources in a utility-maximizing way, it may, nevertheless, have a comparative score in at least one academic area that is low and a comparative score in at least one other academic area that is high relative to that for other individual schools or school districts to which comparisons are made, mutatis mutandis.

Suppose that the educational utility functions for two different school groups, $i$ and $j$, are given by different functions, $u_{i}$ and $u_{j}$,

and

$$
u_{i}=u_{i}(q, v)
$$

$$
u_{j}=u_{j}(q, v) \text {, }
$$

each of which are assumed to have the typical properties associated with such functions. By substitution,

$$
u_{i}=u_{i}\left(q\left(r_{q i}\right), v\left(r_{v i}\right)\right)
$$

and

$$
u_{j}=u_{j}\left(q\left(r_{q j}\right), v\left(r_{v j}\right)\right)
$$

The expected allocations that would satisfy the associated conditions for utility maximization for individual school groups, $i$ and $j$, imply the existence of a relatively low comparative score in at least one academic area and a relatively high comparative score in at least one other academic area for each individual school or school district, to the extent that different school administrators of different school groups will have acted differently to maximize different educational utility functions. Note that such individual school or school districts are, nevertheless, each maximizing educational utility, but with respect to different educational utility functions, $u_{i}$ and $u_{j}$, respectively. Even though $u_{i}$ is maximized with $r_{q i}$ and $r_{v i}$ that yield values of $q_{i}$ and $v_{i}$, respectively, the relative positions of $q_{i}$ and $v_{i}$ in a report of comparative test scores is dependent not only on $q_{i}$ and $v_{i}$, but by the values of $q_{j}$ and $v_{j}$ resulting from the choice of other individual school or school district administrator, $j$, beyond the control of the administrator for the individual school or school district, $i$, in question.

Let the values for $r_{q i}$ and $r_{v i}$ result in area test scores, for example, of 41 and 59, respectively, for individual school group $i$. Let the values for $r_{q j}$ and $r_{v j}$ result in test scores, for example, of 53 and 47, respectively, for individual school group $j$. The comparative scores for group $i$ are relatively low in quantitative reasoning and high in verbal proficiency and for group $j$ are relatively high in quantitative reasoning and low in verbal proficiency. ${ }^{9}$ If such values for $r_{q i}$ and $r_{v i}$ and for $r_{q j}$ and $r_{v j}$ exist where utility-maximizing conditions hold, that is, if $q_{i r q}=v_{i r q}$ and $q_{j r q}=v_{j r q}$, for qroups, $i$ and $j$, and if more resources, then, are spent on quantitative reasoning and necessarily less resources are spent on verbal proficiency in $i$ and if less resources, then, are spent on quantitative reasoning and necessarily more resources are spent on verbal proficiency in $j$, the reallocations, in response to the comparative scores across school groups in this case, would have necessarily negative effects on educational utility for both groups.

Any change in the allocation of resources of the type typically suggested on the basis of comparative test scores across school groups would result in less than the maximum educational utilities with respect to such different educational utility functions. A claim to the contrary would violate the original assumption that the initial allocations of resources, from the associated solutions of the three partials of Equation (4), were utility-maximizing. As such, comparative scores for the various areas of testing to which resources are distributed are neither 
necessarily or sufficiently informative for the systematic determination of utility-increasing reallocations of educational resources. The relative position of an individual school or school district is determined not only by their decision to allocate resources to the various academic areas for which comparisons are made, but the decision to allocate resources by other schools or school districts over which they have no control.

\section{Summary and Conclusion}

Is the typical suggestion of change in resource allocation given the report of comparative test scores by academic area necessarily utility-increasing? If different school administrators allocate resources under utility-maximizing conditions, but with respect to different educational utility functions, then even in this case, the students in such schools or school districts would be expected to have comparative test scores that are necessarily low in at least one academic area and high in at least one other academic area, mutatis mutandis. To reallocate educational resources, therefore, on the basis of the existence of comparative test scores can not be expected to be utility-increasing for any individual school or school district, despite the existence of low and high comparative scores in such individual schools or school districts. A claim to the contrary would violate the original assumption that the initial allocations of resources are utility-maximizing. Unless empirical work on the relationship between comparative test scores and resource allocation is undertaken to provide knowledge of comparative marginal utilities by academic area, reallocations of available resources sug-

\section{Endnotes}

\footnotetext{
${ }^{1}$ Such comparative test scores most commonly take the form of percentile scores by academic area derived from absolute scores for comparisons to other individual schools or school districts. Sometimes, however, absolute test scores are transformed into stanines, despite the condition that neither parents nor counselors are likely to be able to provide a definition of a stanine.

${ }^{2}$ Such a question with respect to the results of administrative decisions has long existed and is currently relevant. See K. Leithwood and M. Stager, "Expertise in Principals' Problem Solving," Educational Administration Quarterly, v25, n2 (May 1989), and H. Simon, Administrative Behavior: A Study of Decision-Making Processes in Administrative Organization, 3rd. ed., New York: The Free Press, 1976.

${ }^{3}$ Equation (1) is assumed to be continuous, with first and second partial derivatives that are continuous, and strictly quasi-concave in its arguments. The first partials of Equation (1) are assumed to be strictly positive. Attempts, in general, to affect levels of achievement, in terms of scores associated with the various areas of testing, are not constrained to those that redistribute a fixed quantity of educational resources, but include those that increase educational resources by transfers from non-educational areas and by increases in the educational day and/or year. Nevertheless, given the determination for $\mathrm{R}$ in a more general equilibrium framework, distributions of educational resources are assumed to be constrained to $\mathrm{R}$.

${ }^{4}$ Equation (4) is also assumed to be continuous, with first and second partial derivatives that are continuous, and strictly quasi-concave in its
}

gested by policy makers, educational authorities, and members of the general public will have unknown consequences on educational utility.

\section{References}

Carroll, J. (1963). A model of school learning. Teachers College Record, 64, 723-733.

Denham, C., \& Lieberman, A. (Eds.) (1980). Time to Learn. Washington, D.C.: National Institute of Education.

Fisher, C., Marliave, R. \& Filby, N. (1979). Improving teaching by increasing 'academic learning time'. Educational Leadership, 37, $52-54$.

Karweit, N. (1984). Time-on-task reconsidered: Synthesis of research on time and learning. Educational Leadership, 41, 32-35.

Leithwood, K., \& Stager, M. (1989). Expertise in principals' problem solving. Educational Administration Quarterly, 25, 126-161. doi:10.1177/0013161X89025002003

Levin, H., \& Tsang, M. (1987). Economics of student time. Economics of Education Review, 6, 357-364. doi:10.1016/0272-7757(87)90 019-7

Roberts, R., Schrader, R., \& Harryman, M. (1986). Productive use of time: an attack on declining achievement. Journal of Human Behavior and Learning, 3, 32-40.

Simon, H. (1976). Administrative Behavior: A Study of Decision- Making Processes in Administrative Organization, 3rd ed., New York The Free Press.

Wiley, D., \& Harnischfeger, A. (1974). Explosion of a myth: Quantity of schooling and exposure to in instruction, major educational vehicles. Educational Researcher, 3, 7-12. arguments. The first partials of Equation (4) are assumed to be strictly positive, as well. An analysis over time can be simplified by invoking a composite-resource theorem that states that, if prices of a group of resources change in the same proportion over time, the optimal choice of resources is consistent with the solution implied by the consideration of resources as a single resource.

${ }^{5}$ See H. Levin and M. Tsang, "Economics of Student Time," Economics of Education Review, v6, n4 (1987), pp. 357-364; R. Roberts, R. Schrader, and M. Harryman, "Productive Use of Time: An Attack on Declining Achievement," Journal of Human Behavior and Learning, v3, n3 (1986), pp.32-40; N. Karweit, "Time-on-Task Reconsidered: Synthesis of Research on Time and Learning," Educational Leadership, v41, n8 (May 1984), pp. 32-35; C. Denham and A. Lieberman, co-editors, Time to Learn, Washington, D.C.: National Institute of Education (1980); C. Fisher, R., and N. Filby, "Improving Teaaching by Increasing 'Academic Learning Time'," Educational Leadership, v37, n1 (October 1979), pp. 52-54; D. Wiley and A. Harnischfeger, "Explosion of a Myth: Quantity of Schooling and Exposure to Instruction, Major Educational Vehicles," Educational Researcher, v3, n4 (April 1974), pp. 7-12; J. Carroll, "A Model of School Learning," Teachers College Record, v64, n8 (May 1963), pp. 723-733.

${ }^{6}$ The terms, $u_{r q}=u_{r v}$, represent the 1 st partials of $u$ with respect to $r_{q}$ and $r_{v}$, respectively.

${ }^{7}$ The 2nd-order conditions require that the score transformation function is increasing at the quantities at which the 1 st-order conditions are satisfied. If the educational utility function in terms of $r_{q}$ and $r_{v}$ is a regular strictly quasi-concave function over a domain, the quantities at which the 1st-order conditions are satisfied are unique educational 
utility-maximizing quantities over that domain.

${ }^{8}$ Since $u_{r q}=u_{r v}$ for the $r_{q}$ and $r_{v}$ that would result from the solution of the three partials of Equation (4), $u_{q}$ is less than $u_{\mathrm{v}}$ for the $r_{q}+\Delta r$ and $r_{v}$ - $\Delta r$ that would result with the reallocation of a unit of resources, $\Delta r$, implied by the typical suggestion of change in resource allocation to quantitative reasoning and from verbal proficiency. The condition that $u_{q}$ would be less than $u_{v}$ is implied by the condition that $u_{r q}=u_{r v}$ are both less than 0 . The condition that $u_{r q}=u_{r v}$ are both less than 0 is consistent with the 2nd-order condition for utility maximization that results from the strict quasi-concavity assumption, previously stated. The terms, $u_{r q}$ and $u_{r v}$ represent the 2 nd partials of $u$ with respect to $r_{q}$ and $r_{v}$, respectively.

It is not necessary, however, that the values for $r_{q}$ and $r_{v}$ satisfy the utility-maximizing conditions for the reallocation typically suggested by policy makers, educational authorities, and members of the general public to have a negative effect on educational utility. Indeed, even if the initial allocation of resources is not consistent with educational utility maximization, the typical suggestion of change may not be utility-increasing. In particular, if $u_{q}$ is less than $u_{v}$, the reallocation typically suggested by policy makers, educational authorities, and members of the general public would also have a negative effect on educational utility. And such a condition may exist, despite the existence of the concurrent condition that $\mathrm{q}$ is less than $\mathrm{v}$. It is only such, if $u_{q}$ is greater than $u_{v}$, that the suggested reallocation may be utility-increasing. However, even if $u_{q}$ is greater than $u_{v}$, the suggested reallocation is not necessarily utility-increasing. The sign of the change in educational utility for the typical suggestion of change in resource allocation would depend on the magnitude of the suggested reallocation. As such, test scores for the various areas of testing to which resources are distributed are neither necessarily or sufficiently informative for the systematic determination of utility-increasing reallocations of educational resources.

${ }^{9}$ Such an assignment of scores is consistent with the existence of the conditions referenced above. 